\title{
Particular Solution of Cutting Tool Path Applied on Helical Surface with Circular Profile
}

\author{
Tadeusz NIESZPOREK, Piotr BORAL, Rafał GOŁĘBSKI
}

\begin{abstract}
The helical surface with circular profile is a special case of the torus-derivative surface, which is shaped using a rotary tool with the circular axial profile of the tool action surface. The helical surface is machined with a tool positioning, in the characteristic section, has a circular profile, owing to which the accuracy of its execution can be easily checked. This has been proved using consistently the power-law series method. A helical surface equation has been introduced in the form of an explicit function without the envelope condition.
\end{abstract}

Keywords: characteristics; circular profile; helical surface; rotary tool

\section{INTRODUCTION}

Worm gears find wide application in many mechanisms and devices $[1,2]$. In the case of cylindrical worm gears, cone-derivative worm surfaces predominate, which are shaped using rotary tools with a rectilinear action surface profile in the axial section $[3,4,5]$. More and more often, universal numerically controlled CNC machine tools and milling cutters with replaceable sintered carbide inserts are used for machining the worm helical surface $[6,7]$. The machining of heat treated materials by milling is intensively developing, which is driven by economical (for replacement of grinding process with machining) and ecological (easy chip removal) considerations [8, 9, 10]. The specificity of hobbing results in the axial profile of the cone-derivative helical surface not being rectilinear [11, $12,13]$, which makes the verification of worm machining accuracy difficult. Worm wheel machining hobs to be mating with the cone-derivative worm in the worm gear, on the other hand, are geometrically complex and technologically difficult to execute [14, 15].

Tests of machining helical surfaces with a curvilinearprofile tool, for example, shaping torus-derivative helical surfaces using tools with a circular profile in the tool action surface axial section, have been conducted for a long time [16-18]. Helical surfaces can be machined with either a finger-type, disc-type, cup-type or ring-type tool $[1,12$, 19]. A special case of the torus-derivative helical surface is the helical surface F-II $[18,19]$. The helical surface F-II is the envelope of a spherical surface in its motion around the axis of the surface being machined. By contrast, in the case of other mentioned types of tools, it requires a special tool positioning, which increases the capability of the technology of machining of these surfaces [20].

In the case of such (disc-, finger-, ring- or cup-type) tool positioning, at which the plane of the axial section of the circular-profile tool action surface is perpendicular to the trajectory of the circular profile centre in helical tool motion, the helical surface F-II is obtained.

The characteristic is the line of the momentary contact of the tool action surface and the machined surface $[1,12$, 19], and in this case, it will coincide with the circular axial profile of the tool action surface. This facilitates the machining accuracy measurement by verifying the accuracy of execution of the circular worm profile in a given characteristic cutting plane. The development of the studies and the increasingly wide use of concave-convex helical surfaces are due to the fact that they exhibit better service properties compared to cone-derivative surfaces [21-23].

The helical surface F-II it characterized in that, it has in measured worm section identical outline with tool acting surface. Worm outline can be easy verified and measured on coordinate measuring machine.

The equations presented as follow the torus-derivative helical surface have been derived and the thesis formulated above has been proved by using the method of expanding a function into a power series [24, 25].

\section{TOOL ACTION SURFACE}

In the case of milling cutters, the action surface of the tool is the locus of tool blade cutting edges [19]. It is assumed that the action surfaces of tools (a grinding wheel or a cutter) are given by parametric equations. A coordinate system $X$ associated with the basic geometric tool elements is introduced and it is assumed that the $X^{3}$ axis is the tool axis. In addition, a coordinate system $u v t$ is introduced, and associated with the calculation point $P$ and it is assumed that the $t$ axis is perpendicular to the tool surface, while the $u$ axis is tangent to the axial profile of the tool action surface.

The initial parametric equation for the action surface of the cup-type tool (Fig. 1a) in the $\underset{1}{X}$ system is as follows:

$\underset{1}{X}(q, \quad \varphi)=\left[\begin{array}{c}\left(r_{x}+\rho \cos q\right) \cos \varphi \\ \left(r_{x}+\rho \cos q\right) \sin \varphi \\ \rho \sin q\end{array}\right]$

where: $r_{x}$ - tool radius to the circular profile centre in the axial section of the tool action surface, $\rho$ - radius of the circular tool profile, $q$ - parameter of circular profile point position, $\varphi$ - parameter of the tool action surface, $X^{*}-$ the subscript identifies the coordinate system, while the superscript identifies the coordinate, $\alpha_{0}$ - parameter of calculation point position on the tool profile.

Whereas, the equation of this surface, after transformations, in the coordinate system $u v t$ with the origin at the calculation point $P\left(q=\alpha_{0}, \varphi=0\right)$ and the $t$ axis oriented along the normal to the surface at this point, 
as expresses in the explicit form of a power series, is as follows:

$$
t(u, v)=-\frac{1}{2 \rho} u^{2}-\frac{\cos \alpha_{0}}{2\left(r_{x}+\rho \cos \alpha_{0}\right)} v^{2}
$$

where: $\alpha_{0}$ - parameter of calculation point position in the tool profile plane; $u, v$ - tool action surface parameters.
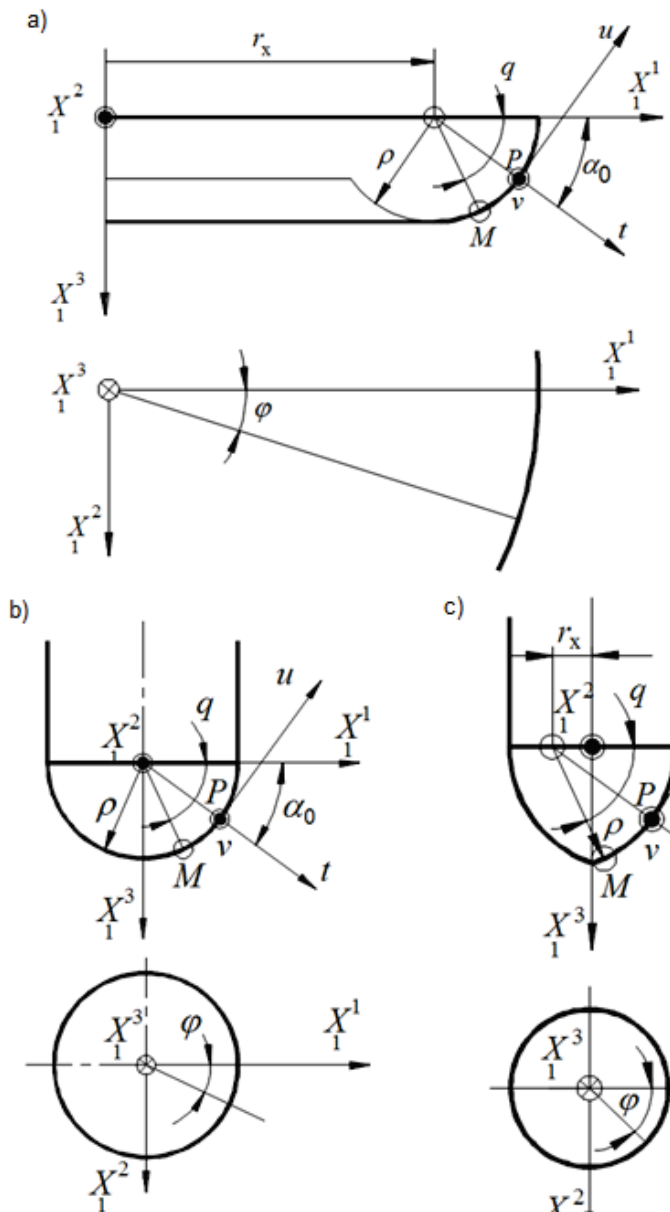

c)

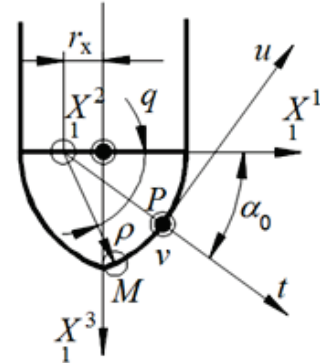

igure 1 The geometry of: a) cup, b) ball-end, and c) finger type tool

Eq. (2) introduces a curvilinear Gaussian coordinate system $u v$ on the tool action surface, whose coordinate lines are, respectively, the curves $u=$ const and $v=$ const. The origin of the $u v t$ coordinate system lies on the surface at the calculation point $P$, while the $u v$ plane is a plane tangential to the surface at this point. So, the direction vectors of the tangents to the surface at this point are tangent to the curvilinear coordinate lines and, as a result, Eq. (2) starts with quadratic terms.

As can be seen from Fig. 1, finger and ball-end tools can be regarded as a special case of the cup tool, and a ballend tool also as a special case of the finger tool. Therefore, the spherical surfaces of the cup, ball-end and finger tools can be described with one general equation in an expanded power series form

$$
t(u, v)=-\frac{1}{2 \rho} u^{2}-\frac{\cos \alpha_{0}}{2\left(k r_{x}+\rho \cos \alpha_{0}\right)} v^{2},
$$

$$
k== \begin{cases}1 & \text { cup tool } \\ 0 & \text { ball and tool } \\ -1 & \text { finger tool }\end{cases}
$$
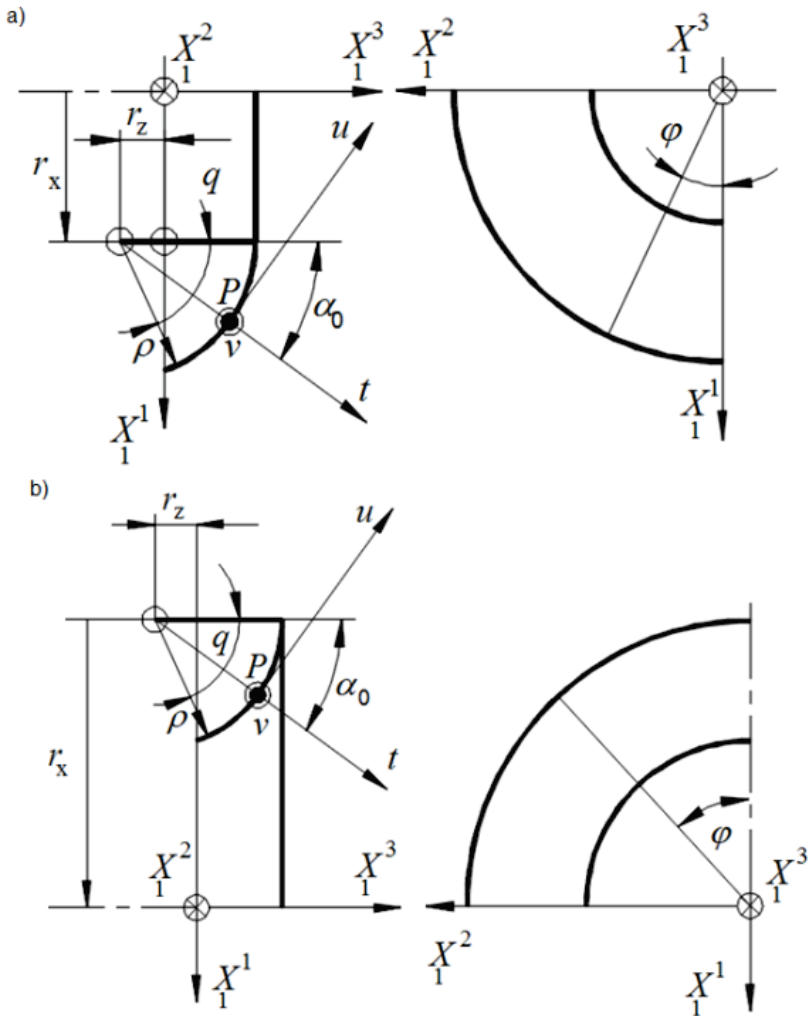

Figure 2 The geometry of: a) disc , and b) rotational tool types

Considering the similar geometry of the tools (Fig. 2), the equations of the actions surfaces of the disc and ring tools in parametric and explicit forms are as follows:

$$
\begin{aligned}
& \underset{1}{x}(q, \varphi)=\left[\begin{array}{c}
\left( \pm r_{x}+\rho \sin q\right) \cos \varphi \\
\left( \pm r_{x}+\rho \sin q\right) \sin \varphi \\
-r_{z}+\rho \cos q
\end{array}\right] \\
& t(u, v)=-\frac{1}{2 \rho} u^{2}-\frac{\sin \alpha_{0}}{2\left( \pm r_{x}+\rho \sin \alpha_{0}\right)} v^{2}
\end{aligned}
$$

where: $r_{z}$ - distance from the circular profile centre to the plane of symmetry of the tool profile in the profile plane, \pm - signs for, respectively, the disc tool and the rotational tool.

Due to the lack of the mixed terms in Eqs. (3) and (5), the coefficients of these equations define the main surface curvatures and, in the cutting plane $v=0$, the tool action surface radius is equal to $\rho$. Eqs. (3) and (5) can generally be written as

$t=\frac{1}{2} t_{u u} u^{2}+t_{u v} u v+\frac{1}{2} t_{v v} v^{2}$

whereas, the coefficient at the mixed term is equal to zero $\left(t_{u v}=0\right)$, which results from the method of assuming the $u v t$ coordinate system. 


\section{THE FAMILY SURFACES}

In the case of shaping a surface by the hobbing method, the moving surface (or the family of surfaces) and the envelope (enveloping surface) contact along the characteristic $[12,19]$. The characteristic is a line that is the locus of the momentary points of contact of the coupled surfaces (the envelope and the surface of the surface family - being mutually enveloping). At the points of the characteristic, the normals to the envelope and the envelope and the surface of the surface family coincide and are normal to these surfaces $[12,19]$. For a sphere, the normals must pass through its centre. If rotary tools (cup, finger, disc and ring types) with a circular profile in the axial section of the tool action surface are taken info consideration, and if the characteristic lies in the axial tool section, then the normals to the tool action surface and the machined surface at the characteristic points will pass through the centre of the circular tool profile. With the relative helical motion of the tool and the machined worm, the machined surface will be the helical surface F-II. In the case of rotary tools, the normals to their action surfaces intersect the tool axis, so the helical surface F-II is in that case shaped by the axial profile of the tool action surface. For the envelope condition to be satisfied with this, the vector of the relative velocity of the envelope and the surface of the surface family must be perpendicular to the tool action surface axial profile plane. So, the tool axial profile must be perpendicular to the helix delineated by the tool action surface circular profile centre in its helical motion. The F-II is in that case shaped by the axial profile of the tool action surface. For the envelope condition to be satisfied with this, the vector of the relative velocity of the envelope and the surface of the surface family must be perpendicular to the tool action surface axial profile plane. So, the tool axial profile must be perpendicular to the helix delineated by the tool action surface circular profile centre in its helical motion.

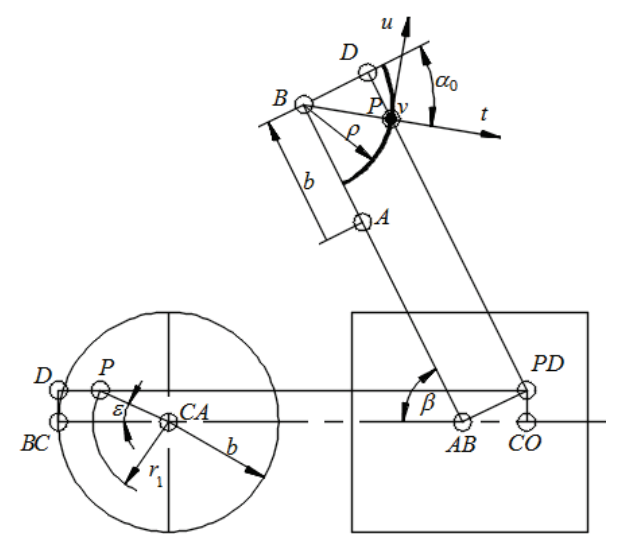

Figure 3 The positioning of the F-II helical surface shaping tool

The $u v t$ coordinate system introduced in a uniform manner for the considered types of the tools described above allows the generalization of the tool positioning pattern for the shaping of the helical surface F-II - Fig. 3.

From the constant worm convolution pitch condition

$h=\frac{2 \pi b}{\tan \beta}=\frac{2 \pi r_{1}}{\tan \beta_{1}}$ where: $r_{1}$ - radius of the worm reference cylinder, $\beta_{1}$ worm convolution lead angle on the reference cylinder, $h$ helical surface pitch, $b$ - distance from the worm axis to the circular tool profile centre, $\beta$ - angle of tool twist relative to the worm axis we can determine the relationship for the radius of the cylinder, on which the helix delineated by the circular tool axial surface centre lies during shaping the helical surface.

$b=\frac{r_{1} \tan \beta}{\tan \beta_{1}}$

Fig. 3 implies, on the other hand, that

$$
b=\rho s \alpha_{0}+\sqrt{\left(r_{1}\right)^{2}-\left(\rho c \alpha_{0} c \beta\right)^{2}}
$$

where the following designations are taken

$$
s \bullet=\sin \bullet, c \bullet=\cos \bullet
$$

where: $s, c$ - abbreviations (operators) to denote the trigonometric functions sine and cosine, for any angle, indicated by a dot $\bullet$.

It follows from the system of Eqs. (8) and (9) that, at the constant helical surface pitch diameter and the constant pitch, with the increase in profile radius the distance $b$ of the tool from the worm axis and the tool twist angle $\beta$ (or the tool positioning) will change.

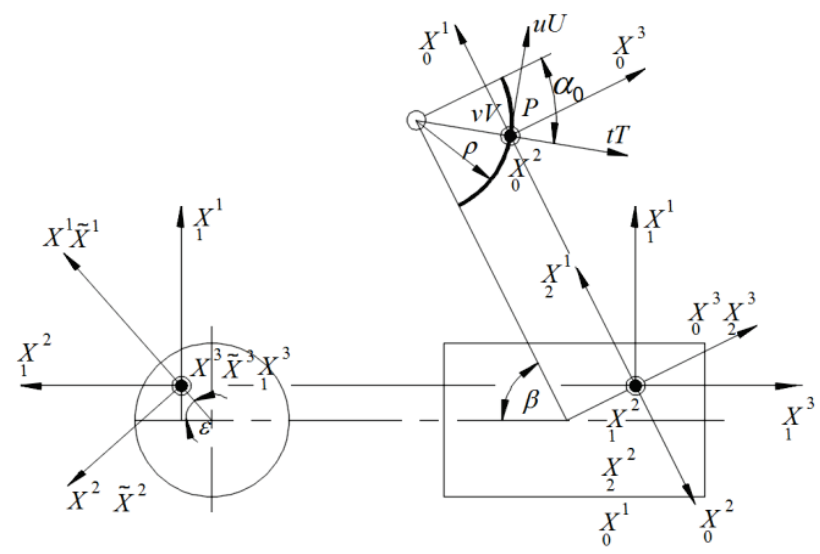

Figure $4 \mathrm{~A}$ diagram describing the relative helical motion of the tool in the machined surface system $(\theta=0)$

To describe the relative tool and worm motion, the worm's system UVT is introduced besides the tool's system $u v t$. At the initial moment, the worm and tool coordinate systems coincide - Fig. 4 .

Because it is hard to describe the relative motion of these systems, an immovable $X$ and a movable $\tilde{X}$ coordinate systems are additionally introduced. The systems $X$ and $\tilde{X}$ coincide at the initial moment, with the $X^{1}$ axis of the $X$ system being perpendicular to the helical surface axis and the second axis, $X^{3}$, being parallel to the former axis. By introducing the additional coordinate systems (Fig. 4), the matrix of transition from the $X$ system to the $U V T$ system is described as follows: $U V T$ 


$$
\begin{aligned}
& {\left[\begin{array}{c}
G \\
U V T \leftarrow X
\end{array}\right]=\left[\begin{array}{c}
M \\
U V T \leftarrow 0
\end{array}\right]\left[\begin{array}{c}
M \\
0 \leftarrow 2
\end{array}\right]\left[\begin{array}{c}
M \\
M \leftarrow 1
\end{array}\right]\left[\begin{array}{c}
M \\
1 \leftarrow X
\end{array}\right]=}
\end{aligned}
$$

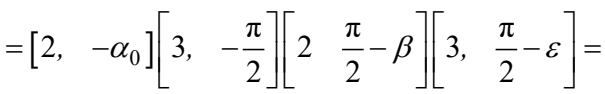

$$
\begin{aligned}
& =\left[\begin{array}{ccc}
-c \alpha_{0} c \varepsilon-s \alpha_{0} c \beta s \varepsilon & c \alpha_{0} s \varepsilon-s \alpha_{0} c \beta c \varepsilon & s \alpha_{0} s \beta \\
-s \beta s \varepsilon & -s \beta c \varepsilon & -c \beta \\
s \alpha_{0} c \varepsilon-c \alpha_{0} c \beta s \varepsilon & -s \alpha_{0} s \varepsilon-c \alpha_{0} c \beta c \varepsilon & c \alpha_{0} s \beta
\end{array}\right]
\end{aligned}
$$

where (Fig. 3)

$$
r_{1} s \varepsilon=\rho c \alpha_{0} c \beta
$$

where: $\varepsilon$ - parameter of calculation point position in the worm's system, Fig. 4).

In the helical surface shaping process, the coordinate systems $u v t$ and $\tilde{X}$ move, while not changing their relative position. As, at the initial moment, they coincided with the systems $U V T$ and $X$, respectively, then the transition between them is also described by the matrix (11). The relative helical motion of the tool and the machined surface, as described by the relative displacement of the coordinate systems $\tilde{X}$ and $X$, can be described by the displacement vector and the matrix of rotation - Fig. 5 .

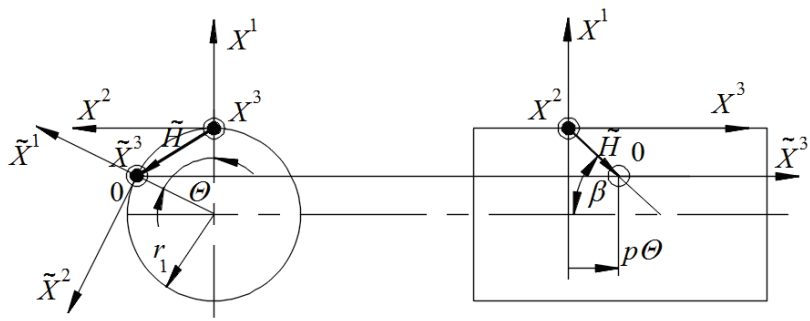

Figure 5 Relative tool and worm helical motion $(\theta \neq 0)$

$$
\begin{aligned}
& {[\tilde{M}]=\left[\begin{array}{c}
\tilde{M}_{X \tilde{X}}
\end{array}\right]=\left[\begin{array}{ll}
3, & -\theta
\end{array}\right]} \\
& \tilde{\boldsymbol{H}}=\left[\begin{array}{lll}
-r_{1}(1-c \theta), & r_{1} s \theta, \quad p \theta
\end{array}\right]^{\mathrm{T}} \\
& p=\frac{h}{2 \pi}
\end{aligned}
$$

where: $p$ - helical surface parameter, $\theta$ - tool and machined surface relative motion parameter.

The notation

$$
\begin{aligned}
& {\left[\begin{array}{l}
k, *] \\
k \in<1,2,3>
\end{array}\right.}
\end{aligned}
$$

means the matrix of rotation by angle $*$ around the $k$ axis.

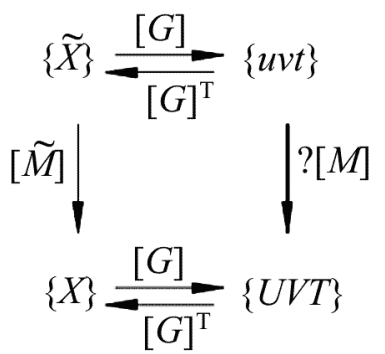

Figure $6 \mathrm{~A}$ diagram of the transformation of the coordinate systems
The displacement vector (14) describes the position of the origin of the coordinate system $\tilde{X}$ in the system $X$. The transition between the coordinate systems $\tilde{X}$ and $X$, as well as between the coordinate systems $X$ and $U V T$, is known, therefore the transition between the systems $\tilde{X}$ and $u v t$ is also known. We need to find the transition between the coordinate systems $u v t$ and $U V T$. We can either make use of the definition of similar matrices or obtain this transformation from the diagram - Fig. 6 .

So, the matrix of transition between the coordinate systems $u v t$ and $U V T$ and the vector of displacement in the UVT system can be written as

$[M]=\left[\begin{array}{c}M \\ U V T \leftarrow u v t\end{array}\right]=[G][\tilde{M}][G]^{\mathrm{T}}$

$\overline{\boldsymbol{H}}=[G] \tilde{\boldsymbol{H}}$

In order to derive the envelope equation, the derivatives of the quantities defined by relationships (17) and (18) should be determined at the calculation point (for $\theta=0)$, or , respectively

$$
\begin{aligned}
& \left.\overline{\boldsymbol{H}}_{\theta}\right|_{P}=\left.[G] \tilde{\boldsymbol{H}}_{\theta}\right|_{P}=\left.[G]\left[\begin{array}{lll}
-r_{1} s \theta, & r_{1} c \theta, \quad p
\end{array}\right]^{\mathrm{T}}\right|_{P}= \\
& =[G]\left[\begin{array}{lll}
0, & r_{1}, & p
\end{array}\right]^{\mathrm{T}}= \\
& =\left[\begin{array}{c}
r_{1}\left(c \alpha_{0} s \varepsilon-s \alpha_{0} c \beta c \varepsilon\right)+p s \alpha_{0} s \beta \\
-r_{1} s c \varepsilon-p c \beta \\
-r_{1}\left(s \alpha_{0} s \varepsilon+c \alpha_{0} c \beta c \varepsilon\right)+p c \alpha_{0} s \beta
\end{array}\right]
\end{aligned}
$$

The hobbing condition implies that the vector of the relative velocity of the envelope and the surface of the surface family should lie in the plane tangent to these surfaces at the characteristic points, while considering the method of taking the envelope's coordinate system, the following condition must be met

$$
\begin{aligned}
& H_{3 \theta}=\overline{\boldsymbol{H}}[3]_{\theta}= \\
& =-r_{1}\left(s \alpha_{0} s \varepsilon+c \alpha_{0} c \beta c \varepsilon\right)+p c \alpha_{0} s \beta=0
\end{aligned}
$$

or

$$
p=r_{1}\left(\tan \alpha_{0} \frac{s \varepsilon}{s \beta}+\operatorname{ctan} \beta c \varepsilon\right)
$$

After substituting relationship (21) in formula (19), the following is obtained

$$
\begin{aligned}
& \left.\overline{\boldsymbol{H}}_{\theta}\right|_{P}=r_{1}\left[\frac{s \varepsilon}{c \alpha_{0}}, \frac{c \varepsilon}{s \beta}\left(1-\tan \alpha_{0} c \beta \tan \varepsilon\right), \quad 0\right]^{\mathrm{T}}
\end{aligned}
$$

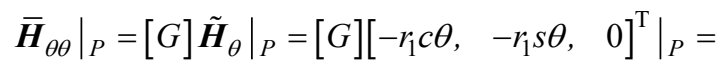

$$
\begin{aligned}
& =[G]\left[\begin{array}{lll}
-r_{1}, & 0,0
\end{array}\right]^{\mathrm{T}}= \\
& =r_{1}\left[\begin{array}{c}
c \alpha_{0} c \varepsilon+s \alpha_{0} c \beta s \varepsilon \\
s \beta s \varepsilon \\
-s \alpha_{0} c \varepsilon+c \alpha_{0} c \beta s \varepsilon
\end{array}\right]
\end{aligned}
$$




$$
\begin{aligned}
& {[\tilde{M}]=[-3, \quad \theta]=\left[\begin{array}{ccc}
c \theta & -s \theta & 0 \\
s \theta & c \theta & 0 \\
0 & 0 & 1
\end{array}\right]} \\
& {\left.[\tilde{M}]_{\theta}\right|_{P}=\left.\left[\begin{array}{ccc}
-s \theta & -c \theta & 0 \\
c \theta & -s \theta & 0 \\
0 & 0 & 0
\end{array}\right]\right|_{P}=\left[\begin{array}{ccc}
0 & -1 & 0 \\
1 & 0 & 0 \\
0 & 0 & 0
\end{array}\right]} \\
& {\left.[\tilde{M}]_{\theta \theta}\right|_{P}=\left.\left[\begin{array}{ccc}
-c \theta & s \theta & 0 \\
-s \theta & -c \theta & 0 \\
0 & 0 & 0
\end{array}\right]\right|_{P}=\left[\begin{array}{ccc}
-1 & 0 & 0 \\
0 & -1 & 0 \\
0 & 0 & 0
\end{array}\right]} \\
& {\left.[M]_{\theta}\right|_{P}=\left.[G][\tilde{M}]_{\theta}\right|_{P}[G]^{\mathrm{T}}=} \\
& =\left[\begin{array}{ccc}
0 & -c \alpha_{0} s \beta & -c \beta \\
c \alpha_{0} s \beta & 0 & -s \alpha_{0} s \beta \\
c \beta & s \alpha_{0} s \beta & 0
\end{array}\right] \\
& {\left.[M]_{\theta \theta}\right|_{P}=\left.[G][\tilde{M}]_{\theta \theta}\right|_{P}[G]^{\mathrm{T}}=} \\
& =\left[\begin{array}{ccc}
-\left(c \alpha_{0}\right)^{2}-\left(s \alpha_{0} c \beta\right)^{2} & -s \alpha_{0} s \beta c \beta & s \alpha_{0} c \alpha_{0}(s \beta)^{2} \\
-s \alpha_{0} s \beta c \beta & -(s \beta)^{2} & -c \alpha_{0} s \beta c \beta \\
s \alpha_{0} c \alpha_{0}(s \beta)^{2} & -c \alpha_{0} s \beta c \beta & -\left(s \alpha_{0}\right)^{2}-\left(c \alpha_{0} c \beta\right)^{2}
\end{array}\right]
\end{aligned}
$$
follows

The coefficients of the surface family equation are as

$$
\begin{aligned}
& t_{\theta u}=M_{31 \theta}-t_{u u} H_{u \theta}-t_{u v} H_{v \theta}=c \beta+\frac{r_{1}}{\rho} \frac{s \varepsilon}{c \alpha_{0}}=0 \\
& t_{\theta v}=M_{32 \theta}-t_{v v} H_{v \theta}-t_{u v} H_{u \theta}= \\
& =s \alpha_{0} s \beta-r_{1} t_{v v} \frac{c \varepsilon}{s \beta}\left(1-\tan \alpha_{0} c \beta \tan \varepsilon\right) \\
& t_{\theta \theta}=H_{3 \theta \theta}-t_{u u} H_{u \theta}^{2}-2 t_{u v} H_{u \theta} H_{v \theta}-t_{v v} H_{v \theta}^{2}- \\
& -2 t_{u \theta} H_{u \theta}-2 t_{v \theta} H_{v \theta}= \\
& =r_{1}\left(-s \alpha_{0} c \varepsilon+c \alpha_{0} c \beta s \varepsilon\right)+\frac{r_{1}^{2}}{\rho} \frac{s^{2} \varepsilon}{c^{2} \alpha_{0}}- \\
& -t_{v v} r_{1}^{2} \frac{c^{2} \varepsilon}{s^{2} \beta}\left(1-\tan \alpha_{0} c \beta \tan \varepsilon\right)^{2}- \\
& -2 t_{\theta v} r_{1} \frac{c \varepsilon}{s \beta}\left(1-\tan \alpha_{0} c \beta \tan \varepsilon\right)
\end{aligned}
$$

The $t_{u u}$ coefficient is identical for all tools, and the $t_{v v}$ coefficient is different - Eqs. (3) and (5).

\section{THE ENVELOPE THE SURFACE FAMILY} follows

The coefficients of the envelope equation are as

$$
\begin{aligned}
& \theta_{u}=-\frac{t_{\theta u}}{t_{\theta \theta}}=0 \\
& \theta_{v}=-\frac{t_{\theta v}}{t_{\theta \theta}} \\
& T_{u u}=t_{u u}+2 \theta_{u} t_{\theta u}+2 \theta_{u}^{2} t_{\theta \theta}=-\frac{1}{\rho} \\
& T_{u v}=t_{u v}+\theta_{u} t_{\theta v}+\theta_{v} t_{\theta u}+\theta_{u} \theta_{v} t_{\theta \theta}=0
\end{aligned}
$$

$$
T_{v v}=t_{v v}+2 \theta_{v} t_{\theta v}+2 \theta_{v}^{2} t_{\theta \theta}
$$

The expressions (34) and (36), with a zero value of the mixed term (35), define the main curvatures of the helical surface, whose curvature radius of the profile in the intersection by the plane $V T$ is equal to the radius of the axial profile of the tool for the machining of this surface, regardless of the type of the tool, provided that its assumed positioning is maintained. The determined surface is the helical surface F-II, whose characteristic section of a circular profile enables the verification of its execution accuracy. In the $U T$ plane, the helical surface curvature radius is described by the expression (36) and depends on the coefficient $t_{v v}$ of the explicit tool action surface equation, and it will be different, depending on the tool type.

\section{EXAMPLE}

A case of determining the helical surface by the envelope method using a disc-type tool is described. In doing so, the positioning of a tool with a circular tool action surface axial profile, designed for machining the helical surface F-II, was taken into account. The geometrical parameters of the tool are the following - Fig. 6: tool profile angle $\alpha_{0}=20^{\circ}$, tool profile radius $\rho=30 \mathrm{~mm}$, tool radius $r_{n}=150 \mathrm{~mm}$, worm pitch radius $r_{1}=100 \mathrm{~mm}$, helical surface pitch $h=360 \mathrm{~mm}$.

For Eqs. (8) and (9), the parameters of tool positioning relative to the shaped helical surface have been determined to be: tool shift $b=109,402 \mathrm{~mm}$ and angular tool positioning $\beta=62,358^{\circ}$.

The axial profile of the tool action surface can be described with the following equation

$$
\underset{z}{\boldsymbol{x}}=\left[\begin{array}{lll}
-\rho s u-r_{x}, & 0, & \pm \rho c u
\end{array}\right]^{\mathrm{T}}
$$

where: $u$ - tool profile parameter.

The tool action surface can be described with the equation

$\underset{d}{\boldsymbol{x}}=[3, \varphi] \underset{z}{\boldsymbol{x}}$

where: $\varphi$ - tool action surface parameter.

Considering relative helical motion of the tool and positioning (Figs. 7 and 8), the equation of the tool action surface family in the worm's system can be written with the equation

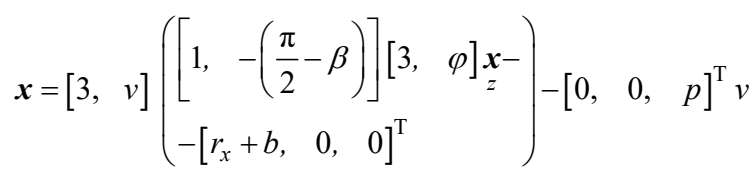

where: $v$ - surface family parameter. 


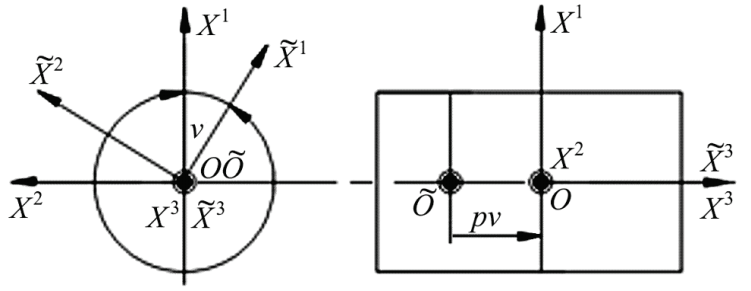

Figure $7 \mathrm{~A}$ diagram of the relative helical motion

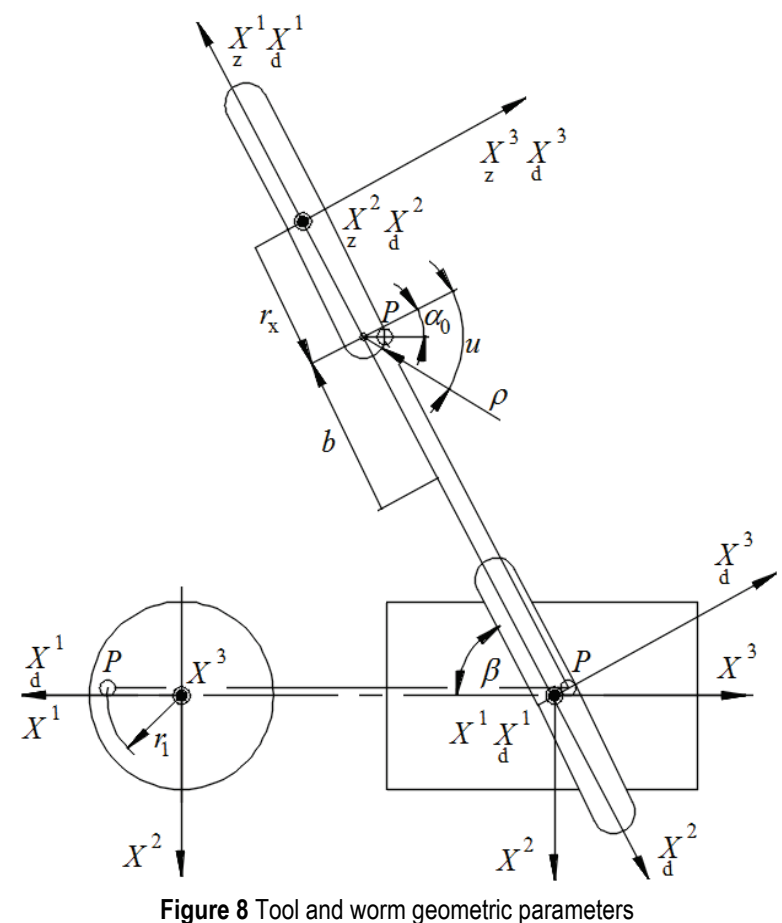

The envelope condition, in the form of the triple product of three vectors $[12,16]$

$f=\frac{\partial \boldsymbol{x}}{\partial u} \frac{\partial \boldsymbol{x}}{\partial \varphi} \frac{\partial \boldsymbol{x}}{\partial v}=0$

leads to the equation

$$
\begin{aligned}
& {[3, \quad \varphi]^{\mathrm{T}}\left[1, \quad-\left(\frac{\pi}{2}-\beta\right)\right]^{\mathrm{T}}}
\end{aligned}
$$

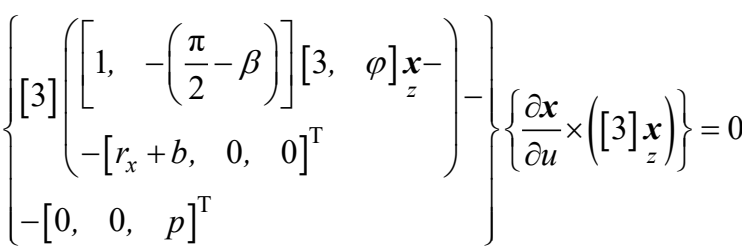

The notation

\section{$[k]$}

$k \in<1,2,3>$

means the matrix of angular velocities [13, 16] (rotation around the $k$ axis).

The helical surface is described by the system of Eqs. (39) and (41). By adding the condition $x^{2}=0$

the worm axial profile has been determined. Whereas, by adding the equation

$\underset{p}{\boldsymbol{x}^{2}}=0$

while

$\underset{p}{\boldsymbol{x}}=\left[1, \frac{\pi}{2}-\beta\right] \boldsymbol{x}$

the worm profile has been determined in the section normal to the lead angle of the helix along which the tool profile centre moves in the worm's system. Whereas, in both sections, the values of parameters $v$ and $\varphi$ have been determined. The values of parameter $\varphi$ are equal to zero, which confirms the above discussion. It also proves that the helical surface F-II can be described by the helical motion of the characteristic (or, in this case, the helical surface FII), by the helical motion of the circular tool action surface axial profile. This means that, in the case under consideration, the helical surface can be described by the equation

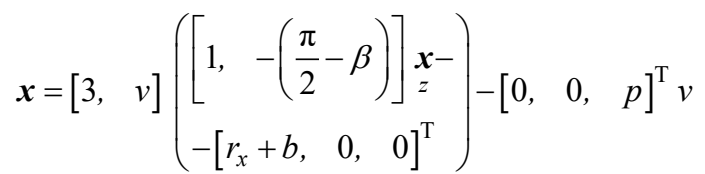

The helical surface parameter, as calculated from relationships (15) and (21), is the same, amounting to $p=$ $57,296 \mathrm{~mm}$.

\section{CONCLUSIONS}

The helical surface profile is circular in the section normal to the trajectory of the tool profile centre in its helical motion relative to the surface being machined. This fact can be utilized for verifying the accuracy of execution of the worm helical surface in that characteristic section.

Therefore, F-II surface stands out among the other helical surfaces. Surface technology is based on this that, axial outline of tool acting profile and worm outline in the measuring section they are identical, which facilitates the worm technology.

In a general case, the radius of the cylinder, on which the tool profile centre trajectory helix lies during helical surface machining, is different from the pitch radius of the machined helical surface. The radius of the cylinder, on which the helix of the trajectory of the circular tool action surface axial profile centre lies, and the angle of grinding wheel twist relative to the helical surface both depend on the magnitude of the profile radius.

The helical surface F-II can be described with the helical motion of the characteristic (the tool action surface axial profile), that is with one equation without the envelope condition (as is the case in the classic method of determining the surface as the envelope of the family of tool action surfaces). 
The power series function expansion method enables the transformation of the parametric equations of the tool action surface into the form of an explicit function and, as a result, the determination of the envelope also in the form of an explicit function without the envelope condition. Expanding the function to second-order terms is generally sufficient in the geometric analysis of machining tools and determination of the machined surface.

\section{REFERENCES}

[1] Dudas, I. (2000). The Theory and Practice of Worm Gear Drives. London: Penton Press.

[2] Marciniak, T. (2013). Worm gear technology. Lodz: Scientific Publishing House of the Institute for Operation Technology.

[3] Litvin, F. L. (1998). Development of Gear Technology and Theory of Gearing. Chicago: NASA Reference Publication 1406.

[4] Dudas, L. \& Dudas, I. (2008). Intelligent Agents Supporting of Design. Type worm gearings. Academic Journal of Manufacturing Engineering, 6(4), 29-35.

[5] Dudas, I. (2004). Investigation of worm gear drive by simulation. Hungary University of Miskolc: $11^{\text {th }}$ International Conference on Tools, 125-131.

[6] Nieszporek, T., Boral, P. (2002). The design and technology of cone worms. The Third International Scientific Conference Mechanics, Rzeszów, 259-264.

[7] Nieszporek, T., Szczepanik, T., Szymański, W. (2008). Manufacturing of Variable-Pitch Cone Worms on Universal CNC Machine Tool. Academic Journal of Manufacturing Engineering. Supplement, 2, 94-100.

[8] Talar, R. \& Stoic, A. (2012). Finish machining of hardened gears wheels using cubic boron nitride $(\mathrm{CBN})$ inserts. Metalurgija, 51(2), 253-256.

[9] Čep, R., Janásek, A., Čepová, L., Petrů, J., Hlavatý, I., Car, Z., \& Hatala, M. (2013). Experimental testing of cutting inserts cutting ability. Tehnički vjesnik, 20(1), 21-26.

[10] Harničárová, M., Zajac, J., \& Stoić, A. (2010). Comparison of different material cutting technologies in terms of their impact on the cutting quality of structural steel. Tehnički vjesnik, 17(3), 371-376.

[11] Radzevich, S. P. (2010). Gear Cutting Tools, Fundamentals of Design and Computation. CRC Press. https://doi.org/10.1201/9781439819685

[12] Litvin, F. L. \& Fuentes, A. (2004). Gear Geometry and Applied Theory. Cambridge University Press. https://doi.org/10.1017/CBO9780511547126

[13] Simon, V. (2004). Precision of worm gear manufacture. $11^{\text {th }}$ International Conference on Tools, Hungary University of Miskolc, 75-80.

[14] Simon, V. (1993). Hob for worm gear manufacturing with circular profile. International Journal of Machine Tools \& Manufacture, 33, 615-625. https://doi.org/10.1016/0890-6955(93)90096-D

[15] Gyenge, C. \& Kismihaly, I. (2000). Minimising of profile errors of hobs for worm gear machining. Annals of DAAM for 2000 \& Procedings of the $11^{\text {th }}$ International DAAM Symposium, Vienna, Austria, 175-176.

[16] Borzan, M. \& Sabau, R. Ch. (2003). The calculus of axial profile of cavex worm. Annals of The MTeM for 2003 \& Proceedings of the $6^{\text {th }}$ International MTeM Conference, Cluj-Napoca, 73-74.

[17] Bosansky, I., Kozuch, M., \& Veres, M. (2006). Hobbing as the possibility of C-C gearing production. The $2^{\text {nd }}$ Int. Conf. "Power Transmissions '06", Novi Sad, Serbia \& Montenegro, 267-270.

[18] Litvin, F. L (1962). Novie vidi cilindričeskih červiačnih peredač. Moskva: Mašgiz. (in Russian)
[19] Nieszporek, T. (2013). Cutting tool design and external cylindrical toothing technology. Czestochowa: Press of the Czestochowa University of Technology.

[20] Nieszporek, T. (1986). Methods of machining channel helical surfaces. Mechanik, 8-9, 353-354.

[21] Seol, I. H. \& Litvin, F. L. (1996). Computerized design, generation and simulation of meshing and contact of worm gear drives with improved geometry. Computer Methods in Applied Mechanics and Engineering, 138, 73-103. https://doi.org/10.1016/0045-7825(95)00976-0

[22] Litvin, F. L, Chen, J. S., Lu, J., \& Handschuh, R. F. (1996). Application of finite element analysis for determination of load share, real contact ratio, precision of motion and stress analysis. Journal of Mechanical Design, Transaction of the American Society of mechanical Engineers, 118, 561-567. https://doi.org/10.1115/1.2826929

[23] Lagutin, S., Verhovski, A., \& Dolotov, S. (2006). Technological design of worm gears with localized contact. The $2^{\text {nd }}$ Int. Conf. "Power Transmissions '06", Novi Sad, Serbia \& Montenegro, 177-182.

[24] Ševeleva, G. I. (1999). Teorija formoobrazovanija i kontakta dvižuščihsja tel. Moskva: STANKIN. (in Russian)

[25] Ševeleva, G. I. (1969). Metoda stepenih radov v teorii zubčatih zaceplenii s točečnim kontaktom. Mašinovedenie, 4 , 58-65. (in Russian)

\section{Contact information:}

Tadeusz NIESZPOREK, Prof.

Czestochowa University of Technology

Institute of Mechanical Technologies

Al. Armii Krajowej 21, 42-201 Czestochowa, Poland

Piotr BORAL, PhD eng

Czestochowa University of Technology

Institute of Mechanical Technologies

Al. Armii Krajowej 21, 42-201 Czestochowa, Poland

E-mail: piotrek@itm.pcz.pl

Rafał GOLECBSKI, PhD eng.

Czestochowa University of Technology

Institute of Mechanical Technologies

Al. Armii Krajowej 21, 42-201 Czestochowa, Poland

E-mail: rafal@itm.pcz.pl 